\title{
Modernization of information-measuring systems for grain processing
}

\author{
Vitaliy Fedotov ${ }^{1, *}$, and Sergey Solovykh ${ }^{1}$ \\ ${ }^{1}$ Orenburg State University, 13, Pobedy Avenue, Orenburg, 460018, Russia
}

\begin{abstract}
The paper discusses the basic operation principles of information-measuring systems for optimization wheat grain processing. The quality of grain processing products (cereals, flour, etc.) is influenced by both weather and climatic factors and grinding technologies. The modern development of information technologies makes it possible to modernize the existing information-measuring systems for grain processing and create new ones through the development of algorithms for analyzing the physical characteristics of the grain mass. During the study, test grinding of wheat grains of different varieties was carried out in a laboratory mill. To increase the yield of the finished product, digitalization of the selection of optimal grain separation modes was used. The obtained mathematical models allow predicting the quality of grain separation in separators of various types. The digitalization of the grain processing industry includes the use of artificial neural networks to analyze images of the grain mass using computer vision algorithms. It is promising to increase the information content of granulometric analysis using modern intelligent (information-measuring) systems. For the classification of wheat according to the milling properties, it is proposed to use the grain hardness. The studies used computer vision and artificial neural networks to find and organize the particles of grain grinding by geometric properties. The characteristics of the contours of the images of the grinding particles were taken into account. The values obtained by the developed information-measuring system were compared with that obtained using the Russian State Standard GOST methods. The error in assessing the grain hardness by the new method does not exceed $3.5 \%$. The use of modern information tools allows improving the quality of wheat grain processing.
\end{abstract}

\section{Introduction}

In the European Union countries, the grain crops' growing essentially depends on the weather conditions. For example, in 2020, 9.65 million tons of grain was harvested in the UK. This is $40.5 \%$ less than in the previous year, due to the reduction in winter wheat plantings by farmers after the wet fall of 2019. Also, the low wheat harvest was exacerbated by the subsequent dry spring. Wheat harvest in Russia in 2020 amounted to about 88 million tons. In the next five years, Russian agrarians will expand the sown area by $40 \%$ by introducing new varieties of wheat and modernizing production technologies. This will increase the yield of durum wheat to 2 million tons per year. Stimulation of durum wheat production will be supported by subsidies at the federal level, a corresponding draft regulation has been prepared for the allocation of subsidies for the production of such grain [1].

Since more than $40 \%$ of spring durum wheat is cultivated in the Orenburg region, in 2020 the government of the Russian Federation decided to conduct an experiment on its territory, gradually expanding the sowing area of this high-quality grain for the needs of the food industry - up to 400 thousand hectares. [2] Durum wheat is preferably grown in hot and dry climates. At the same time, such wheat is more sensitive to soil droughts than soft grain varieties. Of all the districts of the Orenburg region, such conditions are most fully satisfied by the Eastern zone. [3] The yield of durum wheat in the Orenburg region in 2020 amounted to 268.5 thousand tons, which amounted to $10.5 \mathrm{c} / \mathrm{ha}$ [4].

In addition to weather and climatic factors, the production of large volumes of wheat grain is associated with difficulties in its processing. One of the ways to increase the yield of the finished product is the computerization of the choice of optimal operating modes for grain separators. The computer software is based on mathematical models of technological processes. So, it is very important to develop models of separation processes that could improve production efficiency [5].

The structural-functional-energy analysis of separators of various types made it possible to establish that each of them can be considered as one of the varieties of technical queuing systems. In such systems, random processes occur. It drives the need to analyze the nature of the randomness (uncertainty) of the separation processes [6].

It is promising to use the information and measuring systems during milling of wheat, especially of durum varieties. It will ensure the improved quality of grain

\footnotetext{
* Corresponding author: vital asm@mail.ru
} 
processing products and predicting the quality of produced cereals and flour at early stages. The modern development of information technologies makes it possible to modernize the existing informationmeasuring systems for grain processing and develop new ones through the development of algorithms for analyzing the physical characteristics of the grain mass.

\section{Materials and methods}

The wheat grain of the 14 most common varieties of the Orenburg region was studied, the natural weight varied from 720 to $850 \mathrm{~g}$, moisture content varied from $12.5 \%$ to $14.7 \%$, vitreousness - from $45 \%$ to $100 \%$.

To obtain samples of grain grinding, test grain grinding was carried out in a laboratory mill. $10 \mathrm{~kg}$ of grain for each sample was milled. The grinding was carried out with five torn systems with the selection of the top scalp [7].

The fractographic analysis was performed on a laboratory setup consisted of Sony IMX digital camera, Raspberry $\mathrm{Pi} 4$ mini-computer, which was used for image processing. The image analysis was carried out by the developed software based on the OpenCV technical vision algorithms library running the Arch Linux operating system, Deeplearning4j (one of the free sets for training deep neural networks) [8-11].

\section{Results and discussion}

In the course of experiments, it became clear that the result of the separation of grain mixtures in many separators is determined by two kinds of randomness. One of them (mass) is caused by the interactions of particles at high concentrations on the separating organ. In this case, some particles obstruct the separation of others (for example, in a sieve opening). Another (individual) component of uncertainty manifests itself in the absence of mass component when single particles are separated at an ultra-low concentration of the grain flow. In this case, the result of separation is also unknown in advance, since the position (orientation) of the elongated grain in the separating element is random (for example, in a sieve opening).

There is a deep relationship between these components of randomness. It manifests itself with a gradual increase in the separation performance from ultra-low values to the real ones when the separation result initially depends on only one individual component with a gradual increase [12].

The existing approaches for separation processes' modeling consider either only the individual component (classical models of single caryopses motion through the working bodies) or only the mass component (kinetic models of separation processes). So, in real conditions, the models of the first type overestimate the separation result (the influence of the mass component is not taken into account), and the models of the second type underestimate it (the influence of the individual component is not taken into account) [13].
To improve the accuracy of forecasting the separation results, it is proposed to simulate these processes using the apparatus of the queuing theory. Within the framework of this approach, the probabilistic result of separation naturally takes into account the influence of both components [14].

When creating models of separating queuing systems, it is necessary:

- To present the working body in a form of a parallelsequential system of separating elements (cells of triers, sieve openings, elementary volumes of the air channel, etc.), which will act as channels (devices) of the separating systems;

- To express the intensity of the input and yield grain flows through the technological characteristics of the separator, in particular through the productivity;

- To determine the discipline of servicing (separation) of grain particles (applications) in the elements of the working body: sequence, degree of reliability, patience, priority, etc.;

- To describe the possible states of the working body (there is not a single caryopsis in the service; one caryopsis; two; three, etc.).

The model determined in this way makes it possible to obtain equations for calculating the probabilistic characteristics of the separating system using standard methods for the queuing theory.

The study has shown that the result of separation naturally depends on the individual and mass components of the randomness of the separation process. Moreover, it predicts the existence of two groups of separators, one of which admits the possibility of separation by one particle, the other does not. The first group includes most of the known types of separators (air, sieve, electric, magnetic, optical, etc.), and the second group includes only disk trier and sieve modules of high intensity, which differ in slightly larger sieve openings than the particles to be separated. In addition, it correctly reflects the known experimental dependences of the separation quality on the specific load and grain flow rate, on productivity, etc.

The obtained mathematical models make it possible to predict the quality of grain separation in separators of various types, in particular of the first group. This requires creating a methodology and finding an algorithm for calculating its components. [15]

For such calculations, it is necessary to study such a state in the model of a separating queuing system when the productivity tends to zero. Physically, this is the case of separation on single particles, and therefore it requires the use of classical models of well-known separators, i.e., such models that are nothing more than the wellknown equations of dynamics. Traditionally, they are considered deterministic. However, these equations are neither deterministic nor stochastic. Their character is determined by the initial conditions of motion, physical properties of grain particles, operating modes of the separating body.

Indeed, if these parameters are fixed, then the particles will receive one single (deterministic) trajectory. If the initial conditions and (or) physical properties of the grain components of the mixtures, and 
(or) operating modes are varied within wide limits, then we get a wide variety of trajectories of grain particles, and some of them fall into different fractions of the separator. This diversity can be considered as numerous realizations of a stochastic process and a confirmation that classical models are degenerate states of a random process occurring in separating queuing systems.

These data sets should be processed by statistical methods. Then, having sufficient computing power, it is possible for separators of various types in any given mode to calculate the probability of the yield of different components of grain mixtures at ultra-low productivity.

Thus, the proposed approach to modeling made it possible for separators of various types to practically calculate (predict) the desired quality indicators (yield and content of mixture components in fractions) at any given operating mode, including productivity.

Based on models of individual separators, it became possible to predict the quality of separation of mixtures in technological lines of grain cleaning and to solve the following optimization problems:

- Cleaning of a batch of grain (the composition and content of the mixture components are known) on a strict technological line (the number of separators, their type, and installation sequence are known) in the specified operating modes of each separator. The model answers whether this mixture will be cleaned according to the requirements of the Russian State Standard GOST and what will be the yield;

- Cleaning of a batch of grain to the requirements of GOST on a strict technological line. The model determines whether all separators in this line should work and in what modes, which fractions are main and what will be the yield, how it exceeds that on the base line;

- Cleaning of a batch of grain according to the requirements of the Russian State Standard GOST on a flexible technological line. The model determines the number, type and sequence of separators in such a line, the main fractions and operating modes of each separator, the yield of cleaned grain, and its excess in comparison with the base line.

Digitalization in the grain processing industry includes methods for assessing the quality of grain according to various physical and chemical indicators. The industrial device Grain Check analyzes the images of the grain mass by artificial neural networks, determining the presence of impurities, the distribution of grain by size and shape. There are methods for assessing the contamination and glassiness of grain using computer vision algorithms.

To increase the efficiency of grain cleaning machines, photoseparation or photocleaning are used for separation of impurities from grain by optical properties. Photoseparation includes high-speed scanning of the grain mass and subsequent image processing according to a predetermined algorithm (distinctive criterion). The grain can be sorted by size, color characteristics, shape, and other characteristics.

Grain raw materials in the stream are evenly distributed in one layer by vibrating feeders, which allows scanning the image of each particle. Particles are selected using air jets (due to a pneumatic ejector), redirecting impurities or substandard grain to the waste tank. Modern methods of photoseparation allow sorting raw materials according to a whole set of properties, individually and in a set, obtaining a product with the highest possible technological properties.

Additional information on the quality of the raw material can be obtained using infrared or ultraviolet light instead of visible. Different areas of grain reflect slightly different wavelengths. Thus, the endosperm (its grinding) is highly reflective in the IR range compared to the embryo. Color analysis can also be used to distribute grain processing products by fractions (by morphological characteristics of grain parts - embryo, endosperm, bran).

The information content of granulometric analysis can be increased through the use of modern intelligent (information-measuring) systems. The optical microscopy studies of milled grain make it possible to take into account not only the size of the particles but also their shape.

The information and measuring system is operated using the developed software "Software complex for assessing the quality of products of the grain processing industry using the results of grain analysis" (registration certificate No. 201666050); "Program for the preparation of grinding batches with specified properties" (registration certificate No. 2017660454); "Software tool for determining the milling properties of grain" (registration certificate No. 2018610816). The information-measuring system was created as $\mathrm{S}$ laboratory setup.

The milling industry often uses the grain vitreousness to classify grain, but this indicator does not fully characterize the milling advantages of wheat of different varieties (genotypic factor). The variability of vitreousness is also associated with soil-climatic, agrotechnical conditions (phenotypic factor). In view of this, for a quantitative assessment of the consistency and strength properties of grain, it is proposed to use the grain hardness. Direct methods for determining this indicator with hardness testers are laborious and therefore are not widely used in practice.

The studies used computer vision and artificial neural networks to find and classify grain particles by geometric properties. The characteristics of the contours of the images of the grinding particles were taken into account. The analysis algorithm is as follows: from the centers of gravity of the figures, segments are selected to the perimeter of the figure, calculated are the weighted average value of their lengths $(A, \mu \mathrm{m})$ and the variation coefficient ( $B$, dimensionless). Joint accounting of indicators makes it possible to predict some technological characteristics of grain quality. Analysis of samples of 14 wheat varieties of the Orenburg region (table 1) revealed significant relationships with the grain hardness $\left(H, \mathrm{~kg} / \mathrm{mm}^{2}\right)$.

This relationship has the following form:

$$
H=0.15 \cdot B+0.28 \cdot A+0.90
$$

When comparing the values obtained using the model with that using the GOST methods, the error of the 
Table 1. Physical characteristics of grain milled products.

\begin{tabular}{|c|c|c|c|}
\hline Grain sample No. & $A, \mu \mathrm{m}$ & Variation coefficient $B$ & Hardness $H, \mathrm{~kg} / \mathrm{mm}^{2}$ \\
\hline 1 & 40 & 0.54 & 12.4 \\
\hline 2 & 35 & 0.27 & 10.9 \\
\hline 3 & 38 & 0.37 & 11.7 \\
\hline 4 & 34 & 0.29 & 10.1 \\
\hline 5 & 49 & 0.43 & 14.5 \\
\hline 6 & 54 & 0.51 & 16.0 \\
\hline 7 & 57 & 0.49 & 15.2 \\
\hline 8 & 51 & 0.38 & 16.9 \\
\hline 9 & 56 & 0.30 & 18.4 \\
\hline 10 & 61 & 0.34 & 12.6 \\
\hline 11 & 42 & 0.28 & 10.4 \\
\hline 12 & 34 & 0.38 & 18.9 \\
\hline 13 & 62 & 0.37 & 18.0 \\
\hline 14 & 61 & 0.35 & \\
\hline
\end{tabular}

described method for assessing the grain hardness was not more than $3.5 \%$.

\section{Conclusion}

The software for the information-measuring system of the technological line for grain cleaning has been developed. It contributed to improving the predictive ability of the mixture separation quality at grain cleaning lines and optimizing the cleaning of a batch of grain in the specified operating modes of a particular separator. The regularities of the formation of the strength properties of wheat grain are derived based on the analysis of grain milling products. A method is proposed for predicting the grain hardness through the use of modern means of informatization (digitalization) of the grain processing industry.

\section{References}

1. E.B. Maghirang, G.L. Lookhart, S.R. Bean, R.O. Pierce, F. Xie, M.S. Caley, J.D. Wilson, B.W. Seabourn, M.S. Ram, S.H. Park, O.K. Chung, F.E. Dowell, Comparison of quality characteristics and breadmaking functionality of hard red winter and hard red spring wheat, Cereal Chemistry, 83 (2006)

2. A.E. Smith, Handbook of Weed Management Systems (1995)

3. P.R. Shewry, Wheat. J. Exp. Bot., 60 (2009)

4. K. Rundgren, O. Lyckfeldt, M. Sjöstedt, Improving Powders with Freeze Granulation, Ceramic Industry (2003)

5. E.J. Souza, J.M. Martin, M.J. Guttieri, K. O'Brien, D.K. Habernicht, S.P. Lanning, G.R. Carlson, L.E. Talbert, Influence of genotype, environment, and nitrogen management on spring wheat quality, Crop Sci., 44 (2004)

6. J. Rosicka-Kaczmarek, B. Makowski, E. Nebesny, M. Tkaczyk, A. Komisarczyk, Z. Nita, Composition and thermodynamic properties of starches from facultative wheat varieties, Food Hydrocolloids, 54 (2016)

7. A.A. Karim, M.H. Norziah, C.C. Seow, Methods for the study of starch retrogradation, Food Chem., 71 (2000)

8. O.K. Chung, J.B. Ohm, G.L. Lookhart, R.F. Bruns, Quality characteristics of hard winter and spring wheats grown under an overwintering condition, J. Cereal Sci., 37 (2003)

9. J.R. Parker, Algorithms for Image Processing and Computer Vision (2010)

10. Y. Pomeranz, Composition and functionality of wheat flour components, Wheat chemistry and technology, 2 (1988)

11. N. Inomata, Wheat allergy, Curr. Opin. Allergy Clin. Immunol, 9 (2009)

12. O. Buyukozturk, B. Hearing, Crack propagation in concrete composites influenced by interface fracture parameters, International Journal of Solids and Structures, 35 (1998)

13. H.-J. Vogel, H. Hoffmann, A. Leopold, K. Roth, Studies of crack dynamics in clay soil: A physically based model for crack formation, Geoderma, 125 (2005)

14. G. Bradsky, A. Kaehler, Learning OpenCV (2008)

15. M. Manley, P. Williams, D. Nilsson, P. Geladi, Agric. Food Chem, 57 (2009). 